
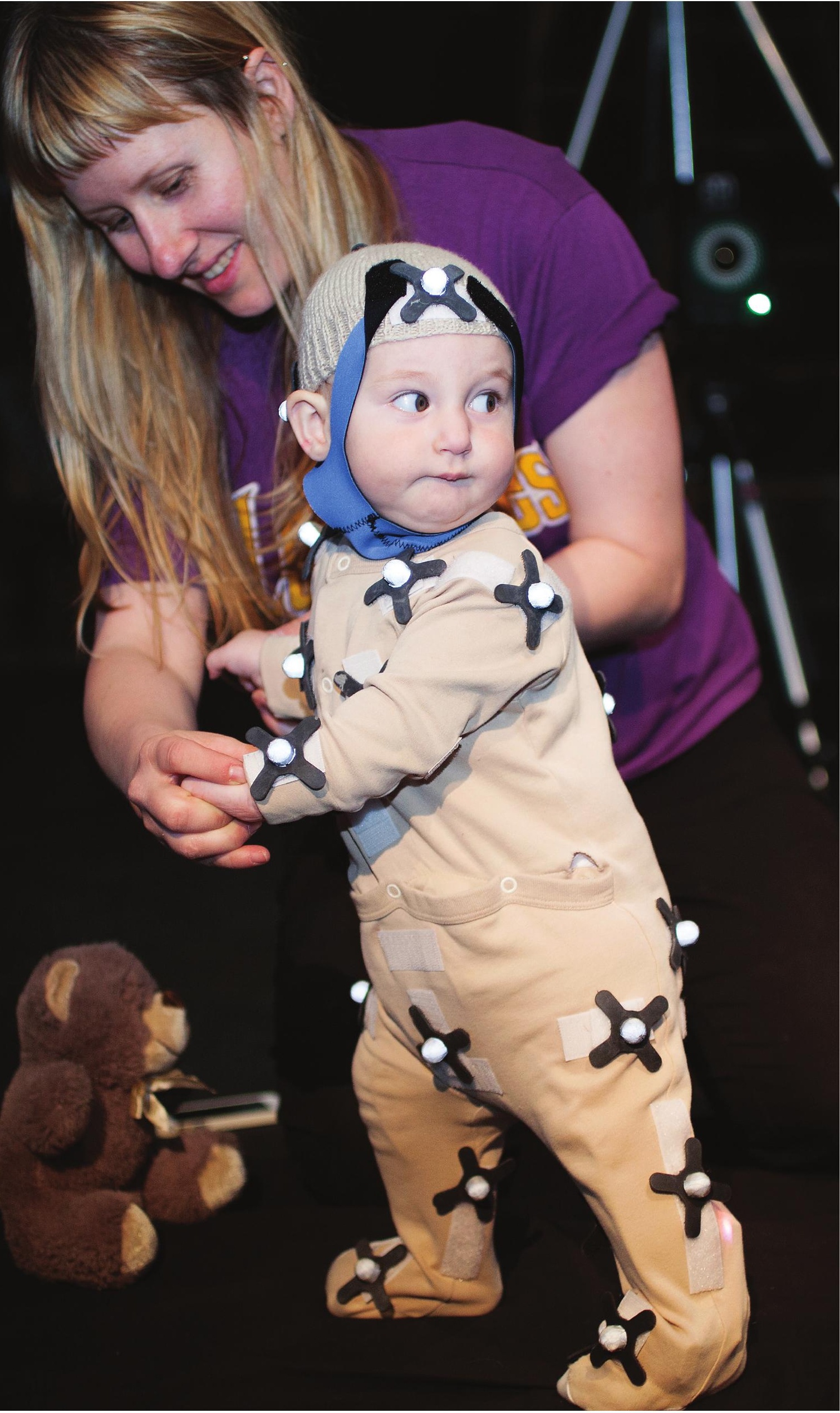


\section{movingstories, Simon Fraser University}

As told by Thecla Schiphorst and Philippe Pasquier
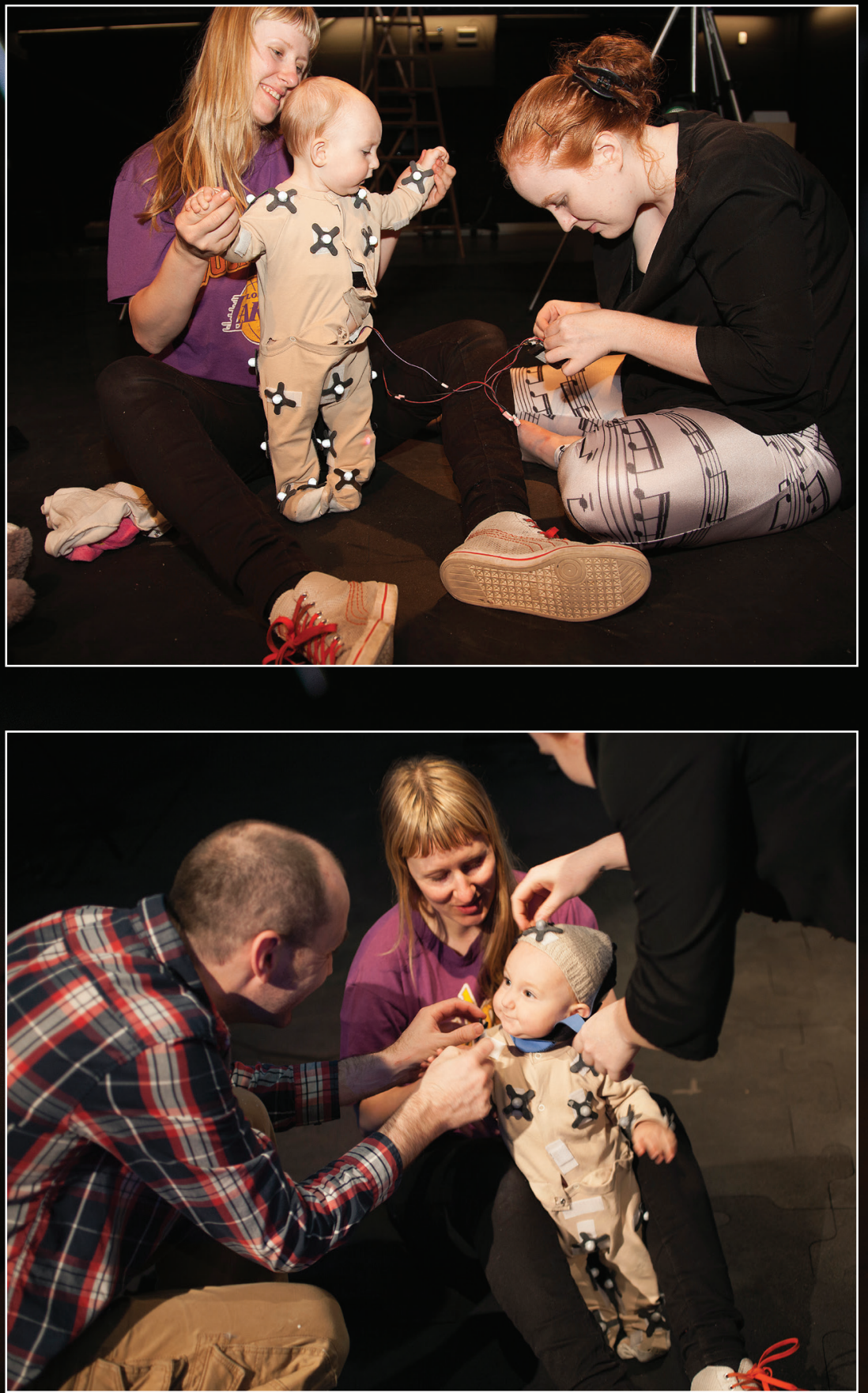

The Baby Owl project examines infant movement development patterns through a longitudinal motion-capture exploration of the movement of one researcher's infant daughter, whose name is $0 \mathrm{wl}$.

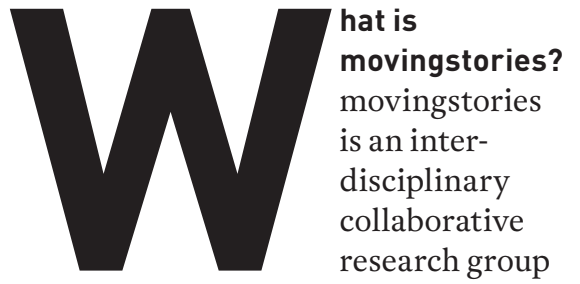

that focuses on movement within technology design.

We strive to create new practices, models, and systems that can lead to the design of digital technologies that incorporate more richly articulated human movement knowledge, such as digital tools for movement, meaning, and interaction. We intersect three unique domains of practice: body-based disciplines such as Laban Movement Analysis (LMA), digital interaction technologies, and social and cultural contexts. We are interested in how movement practice and its foundation in the experience of lived bodily activity can inform technology design. We focus on the design of movement interaction, incorporating computational models of movement recognition, generation, and composition.

movingstories is a collaborative network of studio and black box spaces, a "co.LAB.oration" that inhabits shared "neighborhood" spaces across our research partnership; one might say we share "squatting rights" between our sister institutions.

Our spaces include design studios, a black box performance space, a wearable technology DIY space, and sound studios at SIAT in SFU; dance studios at LIMS and SCA in SFU; a spacious motion-capture studio space at the Emily Carr University of Art + Design; a number of theaters at the University of British Columbia (UBC); and a supercomputing prototyping space at UIUC in Champaign Urbana, Illinois.

\section{What is a unique feature of} movingstories?

In our research...we move! And we highlight movement practice. One of our contributions to movement research is to have certified movement analysts (CMAs) working alongside machine learning and artificial intelligence researchers to bridge technical knowledge across body practices and computational modeling.

We organize ongoing weekly workshop events that support our interdisciplinary approach. A workshop 

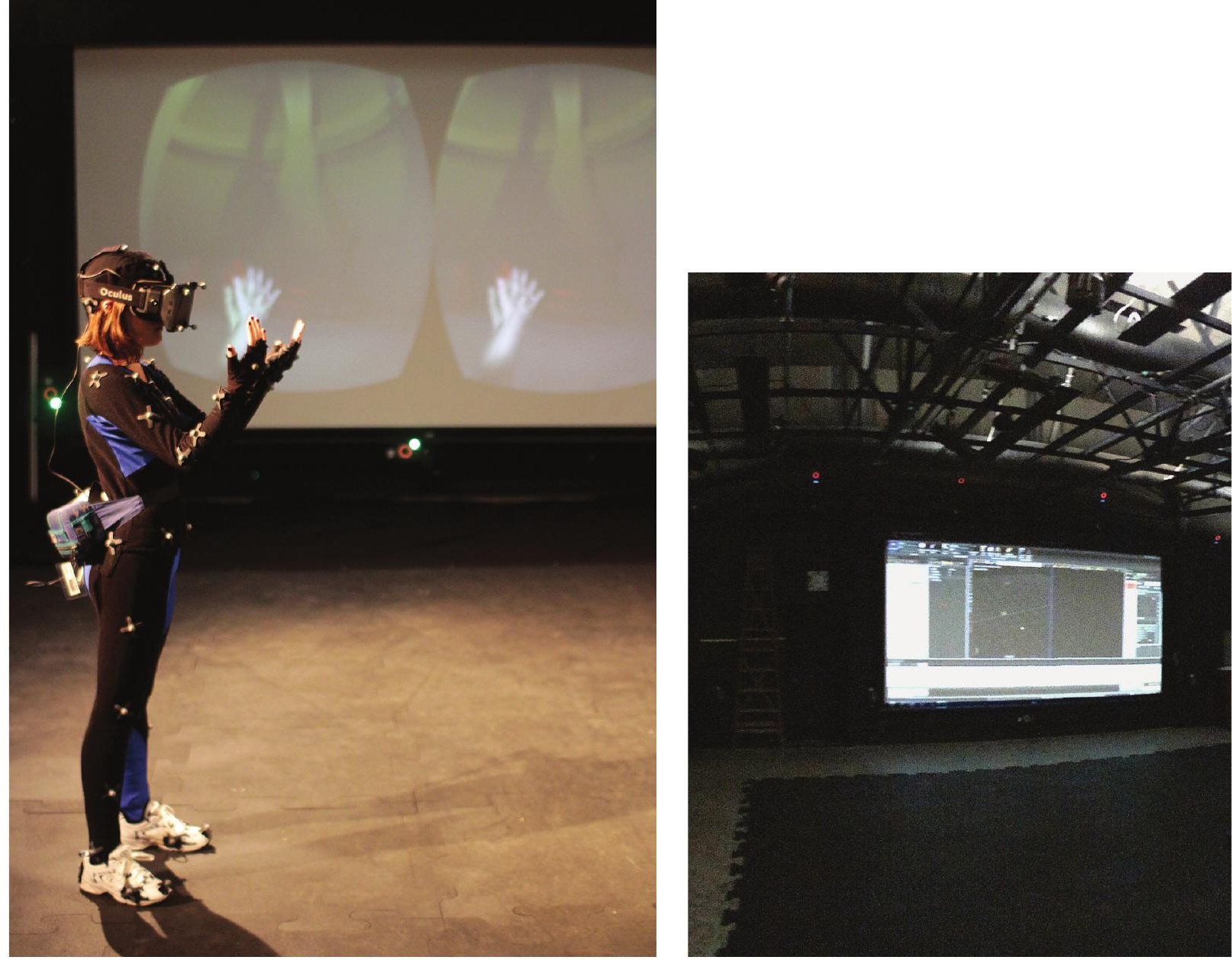

can span two hours or seven days. One such annual event is the week-long May Residency, which includes invited researchers, morning participatory movement activities, afternoon demonstrations, design charettes, and bodystorming activities to set goals, define outcomes, and focus our research objectives.

How do researchers interact and what is the mix of backgrounds and roles? movingstories includes a rich interdisciplinary group of researchers. With six academic institutional partners (SFU, ECUAD, UBC, UIUC, LIMS, CRIM) and three Industry Partners (H+Technologies, Credo Interactive, NZ Technologies) across three geographically separated cities and more than seven research spaces, movingstories includes more than 40 active participants including more than 15 faculty investigators and collaborators, three post-doctoral researchers, and more than 25 graduate students. movingstories continues to attract new connections. We have recently initiated collaboration with researchers at Goldsmith's College in
London and IRCAM in Paris.

Our researchers combine interdisciplinary knowledge domains of movement analysis, compositional theory, design theory, human-computer interaction, artificial intelligence, machine learning, cognitive science, computer games, graphics and animation, and dance history. Among the graduate student researchers there is a vibrant sense of agency, inclusion, and creativity, incorporating film nights and movement sessions that can include yoga, dance, floorwork, contact improvisation, Alexander Technique, or even a simple breathing class.

\section{Could you give some examples of recent projects?}

The creative collaboration between science and art remains a vital locus in movingstories. Our current research includes:

- recognition and generation of movement qualities, including semantics such as personal movement signatures and movement styles (explored through Laban Movement Analysis [LMA]), that can be applied to movement in the database
- development of an open source movement database for annotating and sharing multiple movement platforms that can be retrieved and accessed through a shared movement API

- development of a mobile choreographic tool named iDanceForms that incorporates camera keyframe input and mobile device affordances to support movement sketching and composition.

Our empirical research includes the role and process of movement observation in kinaesthetic empathy, the role of observation in the design of technology, and reliability and validation studies that explore how CMAs apply Laban Movement Analysis to classify and express movement.

An example of a movingstoriessupported art project is a piece called Longing + Forgetting, an interactive urban screen installation collaboratively created by Matthew Gingold, Thecla Schiphorst, and Philippe Pasquier. In this installation, 12 generative video agents are projected onto an urban building facade moving along paths determined by a series of motion graphs that respond to the features of the building (the movement language was 
$\rightarrow$ Panorama of Motion Capture Studio at Emily Carr University of Art + Design.

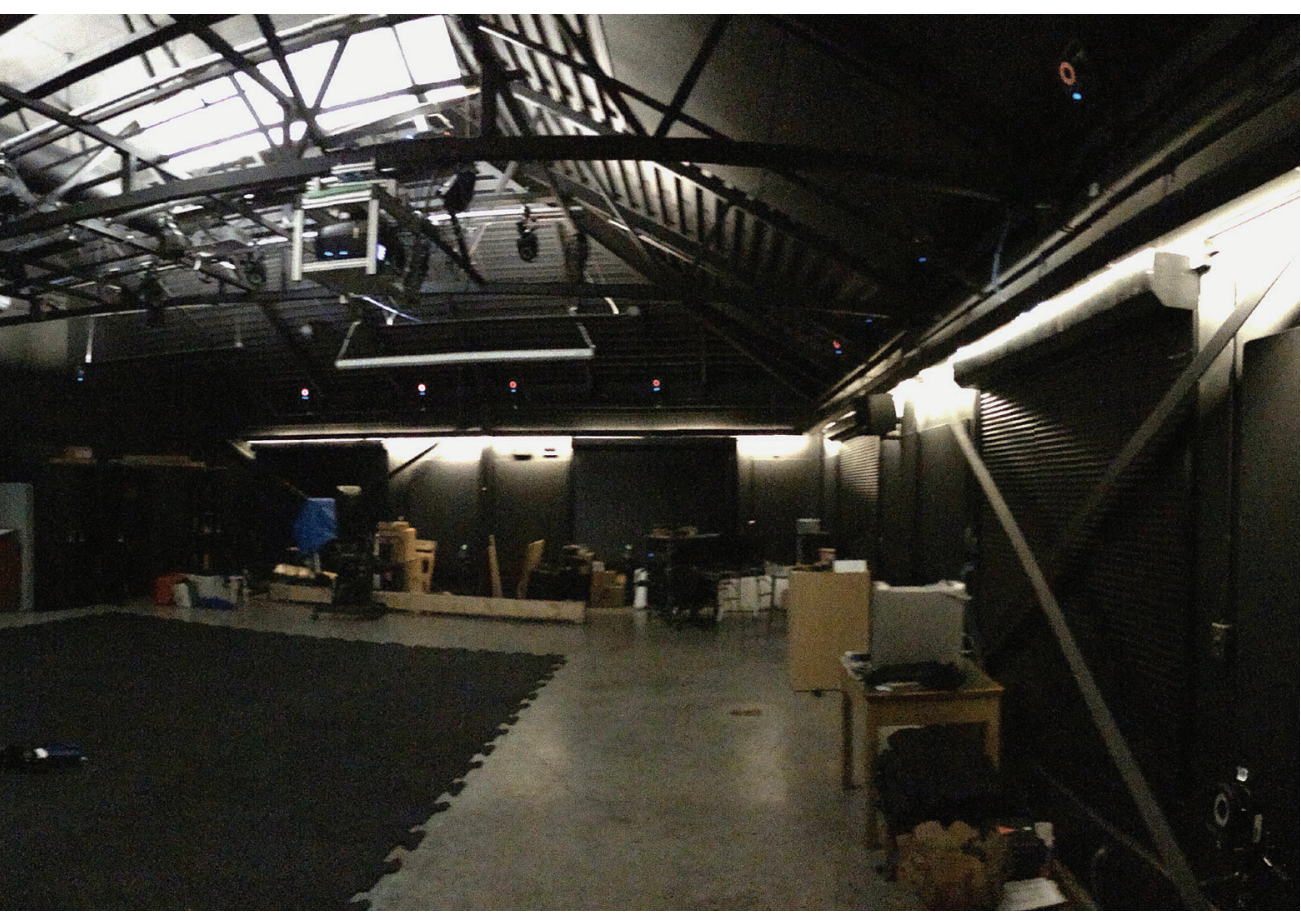

The creative collaboration between science and art remains a vital locus in movingstories. developed from live video footage shot on a specially constructed climbing wall; http://bit.ly/1tFR9Qt). Public participants complete the choreography using the movement of the accelerometers in their mobile phones to activate and interact with the video agents in real time.

\section{What's next?}

While human movement is the soft, messy design material and felt fabric of expression, contemporary technology continues to mediate, explore, and appropriate this design material through computational means.

Our future work in movingstories includes the further description of a movement semantic framework based on articulating experience within computational frameworks. We hope to understand the potency and intelligence of movement as a language for understanding experience, affect, and social context, shaping new methodological strategies for our ever-changing landscape of designing technology and exploring these outcomes in emerging artistic and design contexts.

www.movingstories.ca

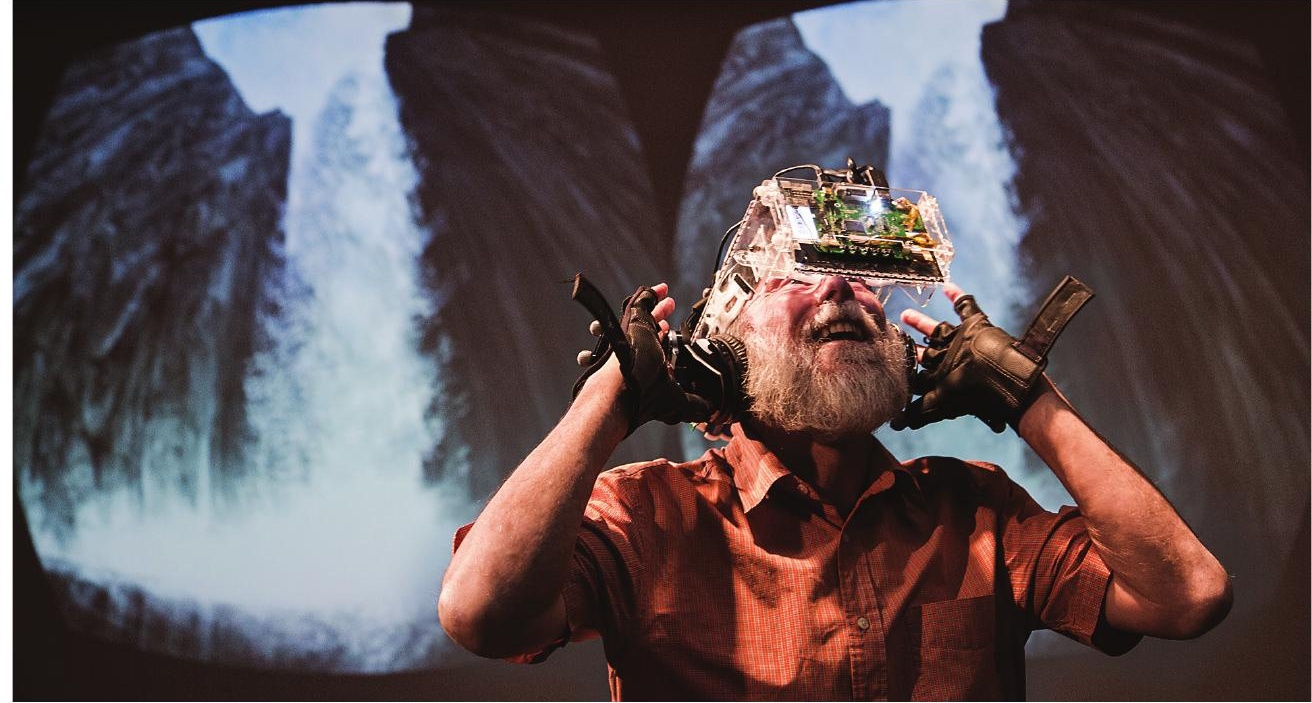

$\rightarrow$ Experiencing movement through the lens of a handcrafted prototype wireless HMD (WaveSine Solutions).

$\rightarrow$ Alexa Mardon performs movement used to correlate kinesthetic knowledge with attention and observation. 